\title{
Computerised determination of spontaneous inspiratory and expiratory times in premature neonates during intermittent positive pressure ventilation. I: a new technique
}

\author{
J N A Mockridge, J S Ahluwalia, C J Morley
}

\begin{abstract}
A pneumatic capsule applied to the sub-xiphisternum provides a signal of spontaneous respiratory effort. Purposewritten software analyses this waveform to determine spontaneous inspiratory and expiratory times. Algorithms reject artefacts. The computerised system allows continuous measurement of spontaneous inspiratory and expiratory times to be made during intermittent positive pressure ventilation.

The accuracy of the system was measured in 17 infants ventilated for respiratory distress syndrome: median (range) gestation and birth weight were 31 (25-38) weeks and $1195(506-3260) \mathrm{g}$. The new technique can measure inspiratory time of individual breaths with a mean error of $5.4 \%$ and expiratory time with a mean error of $1 \cdot 6 \%$.
\end{abstract}

(Arch Dis Child 1994; 71: F156-F160)

Most unparalysed pre-term infants continue to make spontaneous respiratory efforts when ventilated for respiratory distress syndrome (RDS). During intermittent positive pressure ventilation the infant's spontaneous respiratory effort can interact with ventilator inflation and deflation and be associated with adverse outcomes, such as an increased risk of air leaks and intraventricular haemorrhage. ${ }^{1-4} \mathrm{~A}$ means to determine continuously the spontaneous inspiratory and expiratory times ( $\mathrm{Ti}$ and $\mathrm{Te}$ ) during intermittent positive pressure ventilation would be useful in research and may help when selecting ventilator settings. ${ }^{5}$

The ideal technique to obtain such data would be non-invasive, unobtrusive, and allow continuous on-line measurement of spontaneous respiratory times. Many techniques

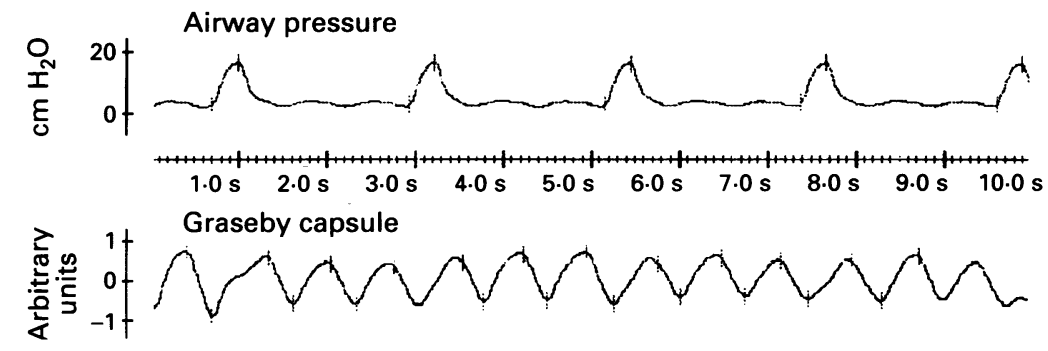

Figure 1 Respiration signal showing little influence from ventilator inflations. The upper trace shows the pressure in the ventilator circuit. The lower trace shows the signal from the Graseby capsule. exist which can be used to measure the respiratory pattern, but they are either too invasive or do not discriminate reliably between ventilator and spontaneous activity. Those that may discriminate but are too invasive for continuous monitoring include the measurement of oesophageal pressure and phrenic nerve activity. Methods which are non-invasive but unable to discriminate spontaneous breaths from ventilator inflations include those that rely on thoracic impedance, plethysmography and measurement of airway pressure and flow.

Measurement of diaphragmatic electromyograms (EMG) is non-invasive and therefore acceptable for use in pre-term infants. However, it is difficult to obtain clear EMG signals because of noise from adjacent cardiac and abdominal muscle activity. ${ }^{6}$

The Graseby pneumatic capsule (Graseby Medical, Berks, England) applied to the sub-xiphisternum provides a reliable signal of spontaneous respiratory activity which is minimally influenced by the ventilator. ${ }^{7}$ Premature infants have highly compliant chest walls and poorly compliant lungs. Diaphragmatic contraction causes xiphisternal retraction. This retraction (clinically seen as recession) distorts the capsule, whereas ventilator inflation leaves the capsule undistorted.

Figure 1 shows a recording where ventilator inflations did not coincide with every spontaneous breath. The signal during spontaneous breaths coincident with ventilator inflation is similar in both shape and amplitude to that without the ventilator inflation. This shows little direct mechanical interference from ventilator inflations on the spontaneous respiration signal.

South et al recorded spontaneous $\mathrm{Ti}$ and $\mathrm{Te}$ in ventilated infants using the Graseby capsule. $^{8}$ However, these infants were all changed over from intermittent positive pressure ventilation to continuous positive airways pressure (CPAP) during the recording, so these $\mathrm{Ti}$ and $\mathrm{Te}$ values do not represent the values during intermittent positive pressure ventilation. Furthermore, these measurements were made for only a few breaths.

This paper describes a computerised system running purpose-written software which gives on-line measurement of spontaneous inspiratory and expiratory times using the Graseby capsule during intermittent positive pressure ventilation. 
Methods

SIGNAL ACQUISITION

The unmodified Graseby capsule is a flat, circular, polythene envelope, $18 \mathrm{~mm}$ in diameter, filled with a disc of polyurethane foam, giving an uncompressed thickness of $3 \mathrm{~mm}$. The capsule has a flange around the foam-filled centre, making the total diameter $28 \mathrm{~mm}$. Trimming this flange reduces the size of the capsule to about $20 \mathrm{~mm}$ and makes it easier to fix securely. This also enhances the differentiation between spontaneous and ventilator breaths. ${ }^{9}$ The capsule is placed on the anterior abdominal wall in the midline with the upper edge just below the xiphisternum. Adhesive tape $1 \mathrm{~cm}$ wide is fixed across the capsule in the midline. Non-compliant tubing connects the Graseby capsule to a pressure transducer with a full scale deflection (FSD) of $\pm 10 \mathrm{~mm}$ $\mathrm{H}_{2} \mathrm{O}$ and a $10-90 \%$ rise time of 1 millisecond (Mercury M9, Mercury Electronics (Scotland) Ltd, Glasgow). The transducer provides an analogue voltage signal corresponding to capsule distortion. Self-zeroing is achieved by incorporating a small leak in the pneumatic system, with a time constant of about five seconds.

The airway pressure is measured at the $T$-piece of the patient circuit (Mercury $\mathrm{M} 9 \pm 300 \mathrm{~mm} \mathrm{H}_{2} \mathrm{O}$ ). The airflow, if required, is measured at the endotracheal tube by a pneumotachograph (Mercury F10L) connected to another transducer (Mercury $\mathrm{M} 9 \pm 10 \mathrm{~mm} \mathrm{H}_{2} \mathrm{O}$ ).

The Graseby capsule signal, the airway pressure, and flow signals are fed into a personal computer via a DT2801 analogue to digital board (Data Translation, Wokingham, Berks), sampling at $50 \mathrm{~Hz}$ per channel.

\section{ANALYSIS BY SOFTWARE}

Purpose-written software, running under MS-DOS version 6.0, displays and analyses the Graseby signal. The airway pressure and flow waveforms can also be displayed on screen. The software algorithms have been developed from the analysis of respiratory traces obtained from recessing neonates requiring intermittent positive pressure ventilation, continuous positive airway pressure, and during free breathing.

The raw respiratory signal is filtered using a seven point moving average (low pass) filter to remove most of the high frequency noise. The filtered respiration signal is then displayed on the screen in real time refresh display. Filtered data are passed to an event detection algorithm which identifies the minima and maxima of abdominal excursions and marks these with vertical lines (fig 1). The Graseby capsule is a movement detector and will detect respiratory and non-respiratory movements. Specific algorithms therefore attempt to identify and reject artefact, leaving only genuine respiratory movements in the calculations. Abdominal excursions are required to conform to two separate constraints, one of amplitude and one of duration, before being accepted as breaths.
The inspiratory times from the previous $n$ accepted breaths ( $n$ is independently adjustable and typically set to 100) are stored in a buffer for averaging. A separate buffer is used for the expiratory times. The median values for $\mathrm{Ti}$ and Te in the buffers at the end of every 10 seconds are output to the screen and stored to disk in ASCII files. The raw data are also stored to disk at the end of each 10 second epoch as binary files.

VALIDATION OF THE TI AND TE GENERATED BY THE SOFTWARE

Validation of any new technique against an accepted gold standard is important. There is, however, no obvious gold standard against which to validate the computerised respiratory analysis system. As described earlier, several techniques exist which can monitor respiratory effort, but none is sufficiently non-invasive and able to discriminate reliably between spontaneous and ventilator activity during intermittent positive pressure ventilation (IPPV). It was therefore impossible to validate this respiratory analysis technique during intermittent positive pressure ventilation. We used a pneumotachograph signal for comparison with the respiratory analysis system during endotracheal continuous positive airway pressure (ETCPAP).

Twenty four infants ventilated for respiratory distress syndrome were studied during brief periods of ETCPAP. Simultaneous recordings were made of the Graseby and flow signals. Both signals of respiratory activity were displayed by the respiratory analysis program.

The respiratory analysis program provides on-screen cursors which can be used to determine the time interval between two selected points to the nearest 0.02 seconds, the sampling interval at a frequency of $50 \mathrm{~Hz}$. The program displays marks on the Graseby capsule signal for the beginning of inspiration and expiration. Different markers are used to indicate whether that part of the recording has met all the criteria for acceptance as a genuine breath. If a breath from the Graseby capsule signal had been accepted and the flow signal was analysable, then values for $\mathrm{Ti}$ and $\mathrm{Te}$ from both signals were measured by aligning the cursors with the appropriate points on each signal.

The onsets of inspiration and expiration were determined from the pneumotachograph signal by identifying the points of zero flow. The values for $\mathrm{Ti}$ and $\mathrm{Te}$ from the pneumotachograph were always determined first. No respiratory signal was excluded that had met the software criteria for genuine breaths, and where the flow signal was analysable.

Table 1 Clinical data on 17 infants whose data were used for validation

\begin{tabular}{lcc}
\hline & Median & Range \\
\hline Gestation (weeks) & 31 & $25-38$ \\
Birth weight (g) & 1195 & $506-3260$ \\
FiO $_{2}$ & $0 \cdot 40$ & $0 \cdot 21-0.95$ \\
Postnatal age (days) & 4 & $0 \cdot 5-11$ \\
Peak inspiratory pressure (cm $\left.\mathrm{H}_{2} \mathrm{O}\right)$ & 16 & $14-24$
\end{tabular}


Table 2 Differences and percentage differences between analysis of flow signal and computerised analysis of Graseby signal during endotracheal CPAP

\begin{tabular}{lll}
\hline & $T i$ & $T e$ \\
\hline Mean (SD) difference (seconds) & $-0 \cdot 02(0 \cdot 06)$ & $0 \cdot 01(0 \cdot 07)$ \\
Mean (SD) percentage difference & $-5 \cdot 4 \%(17 \cdot 0)$ & $1.6 \%(12 \cdot 3)$ \\
95\% CI for the mean difference (seconds) & -0.03 to -0.01 & 0.00 to 0.02 \\
$95 \%$ CI for mean percentage difference & $-7 \cdot 4$ to $-3 \cdot 4 \%$ & 0.1 to $3 \cdot 1 \%$ \\
\hline
\end{tabular}

The difference between the two measurements was calculated for each breath, and the percentage difference for each breath was calculated as follows:

$$
\text { percentage difference }=\frac{2 \times\left(T_{\text {software }}-T_{\text {pneumotachograph }}\right)}{\left(T_{\text {software }}+T_{\text {pneumotachograph }}\right)} \times 100
$$

where $T_{\text {software }}$ and $T_{\text {pneumotachograph }}$ represent values for $\mathrm{Ti}$ or $\mathrm{Te}$ from the software and the pneumotachograph, respectively.

The values for Ti generated by the software from all the infants were combined and treated as one data set and compared with values from the pneumotachograph. The mean values for the differences and percentage differences were then calculated. A similar procedure was carried out for Te.

\section{Results}

Of the 24 infants studied, 17 continued to breathe with an analysable flow signal when changed from intermittent positive pressure ventilation to ETCPAP. Data about the babies are presented in table 1 . The $\mathrm{Ti}$ and $\mathrm{Te}$ measurements are reported for these 17 babies.

A total of 330 breaths were analysable from the flow signals. On the corresponding Graseby signals 266 of these breaths were accepted by the software. Most of the 64 rejected breaths exceeded the software's upper expiratory time constraint. This was due to a transient reduction of the respiratory rate during the change from intermittent positive pressure ventilation to ETCPAP.

Table 2 gives the absolute differences and percentage differences for $\mathrm{Ti}$ and $\mathrm{Te}$ obtained from the flow and Graseby signals. The differences follow a normal distribution. There is a mean difference (95\% confidence intervals) of $-5 \cdot 4 \%(-7 \cdot 4$ to $-3 \cdot 4 \%)$ between the software analysis of the Graseby signal and

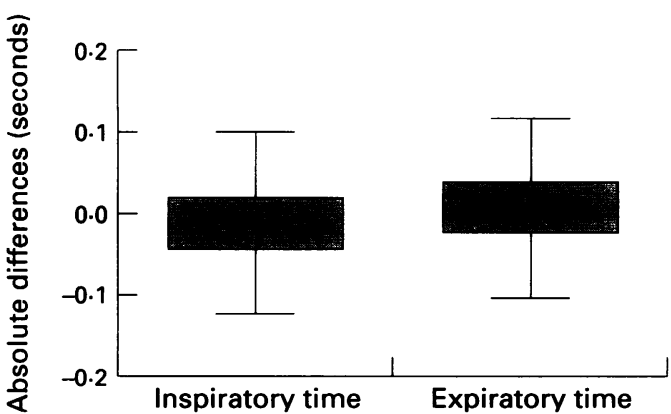

Figure 2 Box and whisker plot of absolute differences between respiratory analysis software and pneumotachograph (software - pneumotachograph) for inspiratory time and expiratory time. The limits of the box represents the interquartile range, the line through the box the median of the differences, with the absolute range denoted by the whiskers. the pneumotachograph for $\mathrm{Ti}$, with a smaller mean difference of $1 \cdot 6 \%(0 \cdot 1$ to $3 \cdot 1 \%)$ for $\mathrm{Te}$.

Figure 2 shows the differences for $\mathrm{Ti}$ and $\mathrm{Te}$ from both measurement methods as box plots. These show that most of the values measured by the software for both $\mathrm{Ti}$ and $\mathrm{Te}$ lie in a range very similar to that for values of $\mathrm{Ti}$ and Te measured by the pneumotachograph.

Figure 3 shows the absolute differences between software and pneumotachograph values for Ti plotted against the average of the $\mathrm{Ti}$ values from each measurement method, according to the approach described by Bland and Altman for assessing agreement between two methods of measurement. ${ }^{10}$ Figure 4 is a similar plot for Te. Most points lie close to zero in both plots, indicating little difference between the two measurement methods, with nearly all the points lying between two standard deviations either side of the mean value. There is no correlation between the values for $\mathrm{Ti}$ and $\mathrm{Te}$ and the size of the absolute difference.

\section{Discussion}

The monitoring of spontaneous inspiratory and expiratory times in ventilated premature neonates is limited by the lack of techniques available that can distinguish between ventilator and spontaneous activity, and that are suitable for clinical use. Previous studies have analysed only short periods of spontaneous respiratory activity. This paper describes a computerised technique to measure continuously the spontaneous inspiratory and expiratory times of ventilated premature neonates with RDS using the Graseby respiration sensor.

Algorithms identify and reject non-respiratory movements based on criteria of both breath amplitude and duration. Due to the large number of respiratory movements available for analysis, the limits for these criteria have been set to ensure that most nonrespiratory movements are rejected, even if some genuine breaths are also rejected as a result. Inevitably there will be some genuine breaths which are interpreted as artefact and some non-respiratory movements which are interpreted as breaths.

The averaging performed on the accepted breaths uses the medians of variable sized lists (or buffers), typically 100 breaths, to reduce the effect of outliers. This has implications for whether changes in the respiratory pattern will influence the calculated values for $\mathrm{Ti}$ and $\mathrm{Te}$, and how quickly these effects would be seen. Large deviations from the current respiratory pattern (with new values which do not fall near the current median), and occurring for a few breaths relative to the size of the lists, would have little influence on the calculated values.

However, large deviations from the current respiratory pattern, occurring for a large number of breaths relative to the list size, would influence the calculated values, but it would take some time for the full effect to become apparent. For example, with buffers of 100 breaths, if a baby has been breathing 


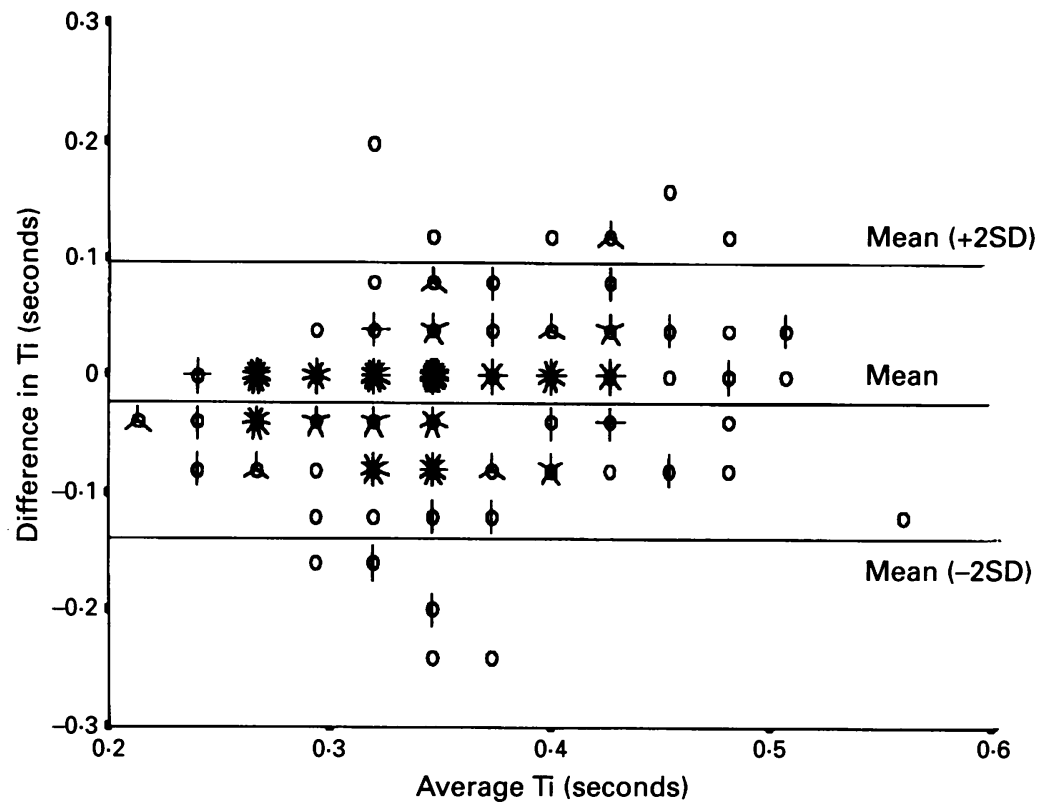

Figure 3 Sunflower plot of absolute differences in inspiratory time (Ti) between software and pneumotachograph (software - pneumotachograph) plotted against the average inspiratory time $\{$ (software + pneumotachograph)/2\}. The reference lines represent the mean difference and two standard deviations either side of the mean.

The circles represent single points. Each additional point with the same coordinates is represented by a line, or 'sunflower petal', so the density of each 'sunflower' indicates the total number of points at those coordinates.

consistently for two minutes with an inspiratory time of 0.30 seconds the inspiratory buffer would contain 100 values each of 0.30 seconds. If the baby then changed its inspiratory time to 0.40 seconds it would take 51 breaths before the new value became the median. At 60 breaths per minute this would take 51 seconds if all the breaths were accepted. If the buffers contained 50 breaths the same change would take only 26 seconds.

In practice the buffers will have a range of values in them at any one time, so that the

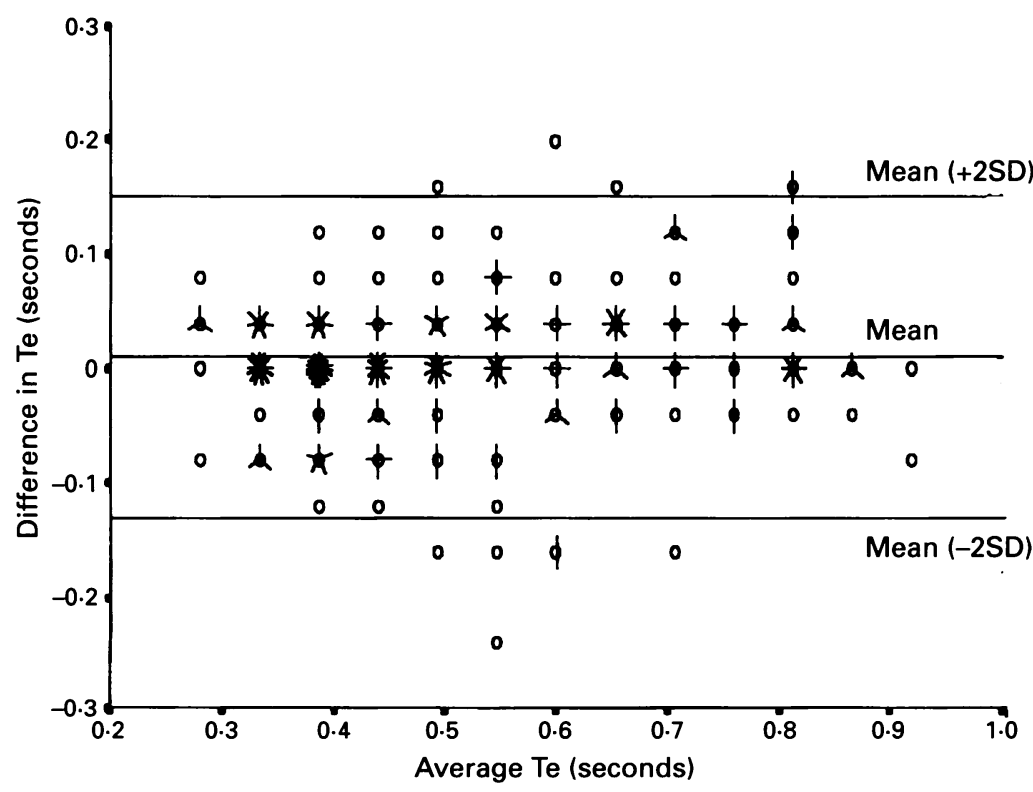

Figure 4 Sunflower plot of absolute differences in expiratory time (Te) between software and pneumotachograph (software - pneumotachograph) plotted against the average expiratory time $\{$ (software + pneumotachograph)/2\}. The reference lines represent the mean difference and two standard deviations either side of the mean.

The circles represent single points. Each additional point with the same coordinates is represented by a line, or 'sunflower petal', so the density of each 'sunflower' indicates the total number of points at those coordinates. new values will have an effect that depends on how long they persist for and where they lie relative to the range of existing values in the buffers. New values close to the current median and within the existing range of values in the buffers could influence the calculated values quickly, regardless of the buffer size.

It has not been possible to validate the technique during IPPV due to the lack of suitable monitoring techniques for comparison. Measurement of oesophageal pressure was undertaken in 10 infants in our study, but the signal was contaminated with peristaltic waves, heart beat, and some of the ventilator pressure which was transmitted to the oesophagus. Validation has therefore been performed during periods of ETCPAP.

Mechanical inflations have little direct effect on the signal from the Graseby capsule when placed on the xiphisternum. ${ }^{7}$ The use of the word 'direct' here refers to the direct mechanical interference of the ventilator inflation on the changes in xiphisternal contour. Intermittent positive pressure ventilation may also change the baby's spontaneous $\mathrm{Ti}$ and $\mathrm{Te}$ from those receiving continuous positive airway pressure, but this influence cannot be determined by this technique and does not affect is validity.

A larger error for the software determination of Ti compared with $\mathrm{Te}$ is not unexpected as it is more difficult to determine the onset of spontaneous inspiration than the onset of expiration. Spontaneous inspiration can start with a gradual increase in inspiratory flow which initially produces little change in the shape of the Graseby capsule and therefore in the corresponding signal. This makes it difficult for the software to identify the exact point at which to mark the respiratory trace for the onset of inspiration. Expiration, however, starts with a sudden flow out of the baby due to the release of the stored elastic energy in the lungs and ribs (as well as possible active expiratory effort), producing a definite and immediate change in the shape of the Graseby capsule. This is easily identified by the software.

The median spontaneous inspiratory and expiratory times of ventilated neonates can now be measured continuously during intermittent positive pressure ventilation. This technique could be used for research into the respiratory pattern of premature infants and their interaction with the ventilator.

\section{Addendum}

An appendix is available from the authors which gives further details of the program structure and algorithms.

We thank the Medical Research Council, British Lung Foundation, Action Research, and the Cambridge Quantum Fund for their support. An international patent application has been filed for this system.

1 Greenough A, Morley CJ, Davis JA. Interaction of spontaneous respiration with artificial ventilation in preterm babies. F Pediatr 1983; 103: 769-73. 
2 Greenough A, Morley CJ, Wood S, Davis JA. Pancuronium prevents pneumothoraces in ventilated premature babies who actively expire against positive pressure inflation. who actively expire 1984 ; i: $1-3$.

3 Perlman JM, McMenamin JB, Volpe JJ. Fluctuating cerebral blood-flow velocity in respiratory distress cerebral blood-flow velocity in respiratory distress
syndrome - relationship to the development of intraventricular haemorrhage. $N$ Engl $f$ Med 1983; 309: 204-9.

4 Perlman J, Goodman S, Kreusser KL, Volpe JJ. Reduction in intraventricular haemorrhage by elimination of fluctuating cerebral blood flow velocity in preterm infants with respiratory distress syndrome. $N$ Engl 7 Med 1985; 312: 1353-7.

5 South M, Morlev CJ. Synchronous mechanical ventilation of the neonate. Arch Dis Child 1986; 61: 1190-5.
6 Chambille B, Vardon G, Monrigal JP, Dehan M, Gaultier C. Technique of on-line analysis of diaphragmatic . . Tur Res $\mathcal{F} 1989$ 2: 883-6.

7 South M, Morley CJ. Monitoring spontaneous respiration in the ventilated neonate. Arch Dis Child 1986; 61: in the

8 South M, Morley CJ. Respiratory timing in intubated neonates with respiratory distress syndrome. Arch Dis Child 1992; 67: 446-8.

9 South M. Observations of spontaneous respiration in ventilated babies [MD Thesis]. London: University of London, 1987.

10 Bland JM, Altman DG. Statistical methods for assessing agreement between two methods of clinical measurement. Lancet 1986; i: 307-10. 